\title{
EDITORIAL
}

\section{A consensus on defining and measuring treatment benefits in dementia}

This issue of International Psychogeriatrics is 96 pages thicker than usual, because it carries not only the International Psychogeriatric Association (IPA) Consensus Statement on Defining and Measuring Treatment Benefits in Dementia, but also a series of papers that were presented at the two-day conference devoted to the development of the consensus statement, which took place in the precincts of Canterbury Cathedral on 31 October and 1 November 2006. During the conference, the delegates (whose names are listed in an appendix to the consensus statement) heard presentations on what outcomes matter to people with dementia and their caregivers, how these can be measured and what they mean, cognitive change as an outcome, biological outcome measures, quality of life, neuropsychiatric symptoms (also known as behavioral and psychological symptoms of dementia (BPSD)), global measures of change, the relevance of different outcome measures to various cultures, activities of daily living, economic outcomes and the regulator perspective. These presentations have been refined into papers and are now published within these pages. In addition to 12 formal presentations on these topics, nine discussants led the conference in exploring the issues raised by the talks, and there was active and often heated debate on almost all the issues discussed. During the afternoon of 1 November agreement was achieved on several key points, which formed the basis for a draft consensus statement prepared the next day. This statement has been refined by a process of circulation among participants, whose suggestions have been incorporated into the document that is now published in this issue.

The importance of dementia as a public health challenge over the next few decades has been extensively rehearsed in this journal and other publications, and the key issues are well outlined in the consensus statement. There is a lot of dementia in the world already and there is going to be very much more in the years ahead. If we are to prevent dementia, modify its course, palliate its symptoms or reduce its impact upon society, carers and people with dementia, we need to agree on what outcomes matter and how they can be measured in different people living in very diverse environments and cultures. As editor of this journal, convenors of the conference and president of IPA, we believe that in organizing the Canterbury Conference and producing the consensus statement,

First published online 9 March 2007. 
IPA, together with Alzheimer's Disease International, the World Federation of Societies of Biological Psychiatry, the World Psychiatric Association and the World Health Organization, has performed a singular service which will benefit dementia treatment research greatly. Ultimately this should be of help to people affected in any way (as patients, family members, friends or as professional caregivers) by this devastating group of disorders.

DAVID AMES

Editor-in-chief, International Psychogeriatrics

Melbourne, Australia

dames@unimelb.edu.au

HENRY BRODATY

Immediate Past President, Alzheimer's Disease International

Sydney, Australia

h.brodaty@unsw.edu.au

EDMOND CHIU

Chair, Section of Old Age Psychiatry, World Psychiatric Association

Melbourne, Australia

e.chiu@unimelb.edu.au

CORNELIUS KATONA

Chair, Old Age Taskforce, World Federation of Societies of Biological Psychiatry Canterbury, U.K.

c.katona@kent.ac.uk

GILL LIVINGSTON

Secretary, Old Age Taskforce, World Federation of Societies of Biological

Psychiatry

London, U.K.

g.livingston@ucl.ac.uk

JOEL SADAVOY

IPA President

Toronto, Canada

j.sadavoy@utoronto.ca 